is due to syphilitic or tubercular ostitis, the temperature has always been practically normal, while in those cases where the cause has been an extension of the inflammatory process from the middle ear to the mas. toid cells, the temperature is usually elevated above the normal at some time during the inflammatory process.

Before closing I wish to mention similar cases which have been published by three American confrères: S. C. Ayers of Cincinnati has reported two cases of the so-called primary abscesses, but admits that there had previously existed an otorrhea, which to my mind excludes these from the cases of primary mastoiditis. Würdemann of Milwaukee has also reported two cases which were thoroughly cured by means of incision and packing. Connor of Detroit reports a case in a child 10 years of age. The drum presented a perfectly normal appearance, and there was no history of a previous discharge. The abscess was thoroughly opened, parts curetted with the final result of perfect healing.

In studying the literature of these cases my conclusions are as follows:

1. Primary abscess of the mastoid is more common than a periostitis.

2. That this pathologic condition is more frequent in children than in adults.

3. The most common causes are syphilis and tuberculosis, and the latter is much more frequent than the text-books would lead us to believe.

4. That the prognosis is nearly always favorable, and a full restoration of the parts is the usual result.

\section{CEREBRAL DISEASE FOLLOWING MIDDLE EAR SUPPURATION.}

Read in the Section on Laryngoiogy and Otology, at the Forty-seventh Annual Meetitus of the American Medical Associatiou. at Atlanta, Ga.. May 5-8, 1896.

BY M. D. LEDERMAN, M.D.

LECTURER ON DISEASES OF THE NOSE AND THROAT, NEW YORK POLYCLINIC: ATTENDING AURAI, SURGEON UNIVERSITY MEDICAL COLLEGE DISPENSARY; ASSISTANT ACRAL SURGKON MANHATTAN EY ND EAR HOSPITAL, ETC. NEW YORK.

Having lately observed three instances of fatal cerebral complications occurring in individuals suffering from suppurative disease of the middle ear, I accept this opportunity of narrating their histories, hoping they may prove of some interest.

When we recall the anatomic arrangement of this cavity, we are impressed with its immediate proximity to vital structures. The partition that separates the middle ear from the brain and its coverings, is but a thin portion of bone, with no diploe. Having little or no illumination, but being sufficiently supplied with heat and moisture, the middle chamber is an ideal incubator for the propagation of pathogenic microörganisms. Diseases of a suppurative character affecting this locality, have many factors to augment their vitality, but comparatively little resistance to limit their spread. It requires no stretch of imagination to picture a purulent inflammation extending through the roof of the tympanic cavity, and attacking cerebral structures.

Observers agree to three forms of pyogenic intraranial invasion arising from middle ear suppuration, i. e., brain abscess, meningitis, and sinus thrombosis. According to Koerner, ${ }^{1}$ almost all brain abscesses, originating from purulent aural disease, are situated near the primary collection of pus, in the ear or temporal bone. Jansen, however, could not discover an extension of the disease from the temporal bone to the brain in any of the cases of abscess of the temporal lobe upon which he had operated, though the collections of pus were directly over the tegmen tympani. To reach them, the posterior and upper wall of the mastoid had to be removed. In 184 instances of intracranial involvement observed by Jansen, in three and a half years, at the Berlin Clinic, 148 were extra-dural abscesses, 35 were thrombosis of the lateral sinus, and only five were brain abscesses. In the extra-dural lesions, the purulent matter was more often found occupying the posterior cerebral fossa. He has observed that extra-dural abscesses are the most frequent complication of acute suppurative otitis media. In cases of doubtful diagnosis he recommends exploratory operations, and further remarks that deep seated collections of pus are best reached after removal of the entire posterior wall of the mastoid process.

It is not always a simple matter to differentially diagnose the presence of existing cerebral disease. When the abscess occupies the motor zone, the direct functional symptoms resulting, assist materially in arriving at a conclusion. If, however, this area is not involved, it requires careful observation to localize the seat of trouble. Picque ${ }^{1}$ remarks, that a persistent cephalalgia, referred to a distinct region, with coma and slowing of the pulse, indicate cerebral suppuration. If the lesion points to an involvement of a motor zone, this locality should be trephined at first, and the mastoid opened later. If on the contrary, indistinct cerebral symptoms arise, the mastoid should be primarily attacked. Should the unpleasant symptoms still persist, further exploration must be carried out.

In endeavoring to illuminate the haze, which at times obscures a positive diagnosis in otitic-cerebral cases, a statement has been made that where bone conduction is present, the abscess is probably situated in the cerebrum; if the bone conduction is absent, the disease is presumptively in the cerebellum. This theory was deduced from the supposition that the pyogenic organisms reach the cerebellum by way of the internal ear. In this manner the infectious process spreading along the auditory nerve and its covering, abolishes bone conduction. Macewan' ${ }^{2}$ claims that as a rule, the cerebellar abscess arises from the disease extending from the sigmoid sinus, and not. from the internal ear, so that the seventh and eighth nerves in the internal ear remain unaffected, and are capable of performing their function. If the mechanism of hearing is not disturbed, the auditory nerve will conduct sound both by air and bone, even though the cerebellar abscess exists. Should, however, the septic process extend to the cranial cavity through. the internal ear, bone conduction may still be present. This fact was demonstrated by a case, reported by Macewan. In this patient auditory conduction was. absent, but bone conduction was more acute on the affected side, than in the normal ear. Postmortem examination revealed extensive purulent leptomeningitis, chiefly marked in the posterior fossa, surrounding the right internal auditory meatus. The seventh and eighth nerves passing through the occluded internal auditory meatus, were swollen and compressed; while on the healthy side, the same nerves occupied. only one-half of its caliber.

1 Annals of Ophthal, et Otology. Vol.iv. No, 4 British Med. Journal 1820-1895. 
Both aerial and bone conduction may be absent without being the result of cerebellar disease. This may occur if some affection of the auditory nerve previously existed. In such a case seen by Macewan, the brain lesion was found to be a cerebral abscess. From his observations he concludes that whether bone conduction be present or not, it is not a reliable sign in localizing the cerebral complication. These contradictory experiences clearly demonstrate that no symptom can be taken as a vade mecum.

Otitic-cerebral abscess is more apt to be the result of a prolonged suppuration and not of an acute affection. Eulenstein, ${ }^{3}$ after collecting authenticated cases, could find but eighteen of brain abscess originating from acute disease of the temporal bone. To this number he adds one of his own, operated upon with a good result. In all these cases the cerebral disease was situated upon the side of the affected bone; the greater number being on the left. Koerner, on the other hand, found the right side to be most frequently attacked. In two of my cases the disease was on the right side. Of the eighteen cases above mentioned, thirteen abscesses were found in the temporo-sphenoidal lobes and four in the cerebellum; the situation of the other one is not given. Among the symptoms observed in connection with cerebral abscesses were: Irregular fever; persistent headache (being one of the most constant symptoms, due to intracranial pressure); retardation of pulse: choked disc; interference with speech (in the left-sided disease); paralyses (facial, hemiopsia, ptosis, abducens on the same side). Other manifestations were. incontinence of urine, photophobia, increased tendon reflex, hemi-anesthesia and hyperesthesia. Eleven patients were operated upon. Of these, five died (two cerebellar, three temporo-sphenoidal); and six were successful (one cerebellar and five temporosphenoidal).

During past years there has existed a tendency to wait for pronounced symptoms before attempting to discover the cerebral complication. Clinic experience has shown us that temporizing under such circumstances is not only unjustifiable but frequently productive of fatal consequences. It matters not in what portion of the economy a septic focus exists, our efforts should be directed toward the prompt limitation of its activity. Accepting this axiomatic principle pro vero we should unhesitatingly attempt to check the stride of an infectious process when it has reached so vital a region. Under present antiseptic precautions, surgical treatment can be undertaken without much fear of external contamination. It is not my intention, however, to sanction hasty and premature operations in these cases, but rather to suggest the prompt employment of effective measures, when indications arise. Suppurative otitis media should not be treated (as is often the case) in a non. chalant manner. Its serious aspect should be vividly pictured to the patient, and the possibility of threatening dangers should be emphasized.

Case 1.-M. C., female, 8 years of age, had a chronic discharge from the ears for three years. Under local treatment the suppuration was checked and no further trouble was noticed. A bout two years after the cessation of the aural trouble the child fell and struck the back of the head with considerable force. At the time of the accident the child was unconscious, and on recovery complained of pain in the head. Three weeks elapsed before any further symptoms appeared. At this time the mother discovered that her daughter was not

3 Monat. für Ohrenheilk. 1895. feeling well and Dr. Charles Hoffman was called. As nothing definite could be found, the condition was treated symptomatically. Irregular temperature became apparent and some pain was complained of in the region of the left ear. Recalling the former otitic trouble, Dr. Hoffman asked me to see the case with him. No local signs of an inflammatory process could be seen. The temperature was 101 degrees F. rectum. No swelling or tenderness of the mastoid or jugular region existed at this time. Treatment was continued as before.

A few days later convulsive seizures together with a somnolent condition of the child was observed by Dr. Hoffman. Applications of ice were ordered to the head and neck. Drs. L. Weber, Hoffiman and myself saw the case in consultation and agreed that meningitis existed, but that the character of the disease could not be definitely diagnosed. As no active ear symptoins were found at this visit, a tubercular manifestation was suspected. Temperature of a septic character persisted and the semi-stupor was rather more marked. Forty-eight hours after our consul tation pressure over the mastoid revealed some tenderness. We decided to operate at once. The usual opening was made under ether. The cortical layer of the mastoid process was found sclerosed, but in chiseling more extensively some pus was discovered. The quantity evacuated was very little. Repeated hypodermic stimulation had to be given while the operation was in progress. The patient's serious condition at this time did not justify further exploration, so the wound was packed with iodoform gauze. On the following morning I trephined an opening into the middle cerebral fossa three-quarters of an inch above the external auditory canal. The dura was bluish in color, but did not pulsate. A hypodermic needle was introduced and about a drachm of pus with drawn. Two more buttons of bone were removed with the trephine, and the connecting bridges of bone were cut away with the rongeur. An incision was then made through the membrane, and fully three ounces of fetid pus escaped. Gentle antiseptic douching was carried out and the parts packed with gauze. As in the previous operation frequent subcutaneous stimulation was found necessary.

Paralysis of the right arm and leg had appeared. Pupils reacted to light in the evening of the day of the second opera tion. Coma continued, paralysis of the sphincters was ob served, and on the following day dissolution occurred.

Case 1 is an illustration of the irregular course a temporo-sphenoidal abscess may run. Though previous ear trouble had existed, no symptoms pointed to this organ at the beginning of the fatal complication. The quantity of pus present in this cerebral abscess demonstrates that such a state of affairs may exist for some time without producing characteristio symptoms. It is possible that the traumatism in this case may have been an exciting factor. The question arises, whether we are justified in opening the mastoid process or cerebral cavity, in cases presenting a similar train of symptoms which give evidence of previous suppurative otitis media, though presenting no active manifestations.

Case 2.-F. M., aged 16 years, white, was admitted to the Manhattan Eye and Ear Hospital, Oct. 5, 1895, with the following history: For the past twelve months had chronic suppuration of the right ear, resulting from the measles. The discharge was not constant, but whenever it stopped severe pain set in, which ceased when drainage was reëstablished. A bout three weeks before his appearance at the hospital, after an exposure, he was taken with severe pain over mastoid, in and around the ear. Pain had been constant since. At this time the external parts were considerably swollen and tender temperature 100 degrees F. ; pressure in front of tragus caused pus to flow freely. Under local treatment the patient progressed nicely until about 2 o'clock A. M., Oct. 6, 1895, when becoming delirious, he was then sent to the hospital.

Condition on admission: Delirious, restless and anxious expression. Temperature 104 F.; pulse 110 . Right mastoid swollen; slight redness extending well down over neck. Marked tenderness over mastoid, front of ear. Decided pulsation in right cervical region. On slight manipulation with probe and cotton profuse offensive discharge came from ear. Meatus perforated in postero.inferior quadrant.

Patient was etherized and a mastoid operation done under strict antiseptic precautions. Small quantity of pus was found in cells. Wound cleansed with bichlorid solution and packed with iodoform gauze. Morphin hypodermic was necessary to quiet during night. 
October 6. Very restless this A. M. Temperature $100 \mathrm{~F}$. pulse 110; complains of sick stomach, but does not vomit; does not take nourishment.

October 7. Temperature $98 \mathrm{~F}$., pulse 110 ; still restless; takes little nourishment.

October 8. Extremely restless: constant tendency to get out of bed. Temperature 99.6 F., pulse 110. Dressing removed. Considerable pus in canal and mastoid. Cleansed and irrigated with bichlorid solution, 1-10000; packed with gauze and bandaged, leaving external auditory canal exposed for douche, every two hours. Morphin to quiet; strychnin and spirits frumenti ordered to be given at the discretion of the house surgeon.

October 9. Still restless; temperature $100 \mathrm{~F}$. Takes nourishment quite freely. Mastoid dressed as before. Wound clean and healing.

October 10. Temperature $98 \mathrm{~F}$, pulse 110. Not quite so restless. Voids urine in bed.

October 11. Temperature $97 \mathrm{~F}$., pulse 120 ; restless; no chills ; stimulants increased.

October 12, $8: 30$ A. м. Some signs of left hemiplegia. Not so restless; breathing slightly stertorous; $9: 30 \mathrm{~A}$. $\mathrm{M}$., will not take stimulants. Died with symptoms of compression at $12: 15$ P. M.

Postmortem examination showed an extensive cerebral abscess of the temporo-sphenoidal lobe. A large quantity of pus escaped after the dura was incised. Four finger tips could be readily introduced into the abscess cavity. The dura was thickened and on the cerebral surface of the tegmen a necrotic area was found. No involvement of the sinus was observed the extension of the disease being by continuity of tissue, and not through the lymphatics.

On Oct. 11. 1895, the patient was doing so well, even though the temperature only registered $97 \mathrm{~F}$., that further operative interference was contraindicated. Had an opening been made on this day into the middle cerebral fossa it certainly could not have saved the patient, as the disease had then assumed extensive proportions.

Case 3.- Phoebe B., deaf mute, 62 years of age, was referred to me at the Manhattan Eye and Ear Hospital, May 6, 1895, for mastoid operation, with the following history :

For past year has had pain in right ear, with scanty discharge. Three months ago, facial paralysis of right side occurred suddenly. The patient had been operated upon for aural polypi, which completely filled the external canal. There was a profuse and very offensive discharge from the right canal, which was somewhat benefited by the removal of the granulation tissue. These growths rapidly reappeared so that repeated attempts at their eradication had to be made.

On May 5 the mastoid process became boggy and very tender. Treatment failed to relieve the pain and swelling, so patient was turned over to me for operation.

May 6, 3 P.м. A Stacke operation was performed, together with the removal of almost the entire tip of the mastoid. The osseous destruction was very extensive, the dura being encountered on passing the probe upward. Thorough curetting of the posterior and inferior surfaces of the diseased tissue was carried out. Fearing perforation into cerebral cavity, gentle manipulation in the upper portion of the wound had to be observed.

May 7. Temperature 99.4 F. Patient doing well.

May 8. Temperature normal. Dressing removed. Some discharge from the canal which was not offensive. Otherwise wound clean. Packed as before and ordered cleansed with peroxid of hydrogen and corrosive sublimate solution.

May 11. Some slough on posterior wall of cavity. Discharge increased, with some odor. The slough was removed and Labarraque's solution ordered as a cleanser.

May 18. Improving; wound clean and rapidly filling. Temperature normal.

May 20. Granulations exuberant. Touched with caustic.

May 25. A small sinus was found on inner wall of cavity, filled with pus; probe showed necrotic bone. Sinus curetted, cleansed, and packed with gauze.

May 30. Improving; wound clean; slight odor from sinus.

June 9. Odor becoming offensive. Some débris curetted from the wound. Inability to move left arm and leg was noticed.

June 11. In the morning, a hemorrhage occurred from the mastoid, appearing through the ear, nose and throat, and saturating the dressings. 7 P.M. Severe hemorrhage through naso-pharynx. Post-nasal tampon applied. Source of bleeding could not be discovered.
June 12. Another hemorrhage, which was checked by tamponing mastoid opening. Hypodermic stimulation.

June 14. Patient gradually grew weaker from the loss of blood, and died at $6: 30$ P.M.

The autopsy revealed marked necrosis of the petromastoid portion of the temporal bone. The probe could be readily passed into the posterior and middle cerebral fossee. Ulceration of the sinus was found to be the cause of the severe hemorrhage. Softening of the right temporo-sphenoidal lobe of the brain was recognized.

Owing to the congenital condition of the patient, subjective symptoms were not readily ascertained. There can be no doubt as to the chronic character of the destructive process, as the facial paralysis had occurred three months previous to the case coming under our observation. So extensive was the disease, that after the operation, a probe could be passed into the mastoid opening for a distance of almost two inches, measured from the external surface.

Case 4.-Though this case can not be positively classed as a cerebral involvement, nevertheless I mention its history for the purpose of emphasizing the difficulty we meet with at times, arriving at a correct diagnosis.

James B., 34 years old, had scarlet fever twenty-five years ago, which resulted in a chronic suppurative otitis media, which has been more or less active ever since. During the past four years the discharge has been more profuse, and frequent attacks of severe pain have been experienced. Relief from the pain was noticed, whenever the discharge flowed freely. Blood was at times found in the discharge. On May 24, 1895, he was admitted to the Manhattan Eye and Ear Hospital, with intense pain in the left ear, from which a scanty flow of pus was observed. Some infiltration of the soft tissues of the external auditory canal existed. A small perforation in the postero-inferior quadrant could be seen on careful inspection. Anterior to tragus, the parts were tender, but no pain or swelling over mastoid.

General condition.--Patient is anemic, and seems to be suffering severely. Tongue heavily coated; bowels constipated; temperature $103 \mathrm{~F}$; pulse 140.

May 25. Bulging of membrane. Tympanum liberally incised. Hot douching every two hours, and boroglycerid tampons were ordered; also calomel, until bowels moved freely.

May 26. Slight improvement. Temperature $101 \mathrm{~F}$. Some headache and pains in back of neck. Leeches applied. No definite conclusion could be reached.

May 30. Temperature still high. Griping pains in bowels calomel stopped. Marked tendency to constipation.

June 1. Great pain in back of neck. Temperature $102 \mathrm{~F}$. Counter-irritation applied.

June 2. Pain somewhat less, but still present. Ice to neck ordered. Bromid was prescribed.

June 4. Considerable pain in neck. Peculiar discharge from bowels resembling that of enteric fever. Temperature 104 F. Our medical consultant' examined the patient, and suspected thrombosis of the lateral sinus.

June 5. Chill lasting twenty minutes. Patient much exhausted. Strychnin and whisky freely administered. Temperature $104.2 \mathrm{~F}$

June 6. Chill lasting fifteen minutes. Quinin and fluid extract digitalis given continuously. Temperature $103 \mathrm{~F}$. Dr. A. H. Smith advised surgical interference. At 8 P.M. I operated. Periosteum was detached from mastoid with great difficulty. The bone was found sclerosed throughout its entire extent. No pus was discovered. On opening into the lateral sinus, nuthing abnormal was found. Sinus and mastoid were packed with iodoform gauze. During the operation patient received hypodermic injections of strychnin.

June 7. Recovered nicely from operation.

June 9. Symptoms of abscess of liver appeared, with great exhaustion.

June 12. Temperature still elevated. Occasional chill followed by pronounced depression, which finally terminated the patient's existence. Unfortunately no postmortem was allowed. We were inclined to believe that the liver symptoms were the result of metastasis. A cranial lesion in the form of a cerebellar abscess was suspected.

We must not overlook the fact that disintegration of osseous tissue may take place, without causing painful symptoms. This is especially characteristic 
of a tubercular process; and in the region of the ear, marked destruction may occur without involving the integrity of the membrana tympani. Such a condition is more apt to affect children of a strumous diathesis. In such instances the ulceration may soften the roof of the tympanic cavity, and so attack the brain. It furthermore may generate miliary tuberculosis of the lung and other organs, by entering the circulation, through the antrum and mastoid cells, or by penetration into the jugular fossa or sigmoid sinus.

128 East 60th Street.

DISCUSSION.

Dr. MAX ThoRner, Cincinnati--The papers are of more than usual interest, because the question of operative interference and topographical relations are comparatively new. Ordinarily topography of the temporal bone is insufficiently treated in text books.

The antrum is ordinarily larger and the floor of it is so much below the level of the aditus that pus can not flow out according to the laws of gravitation. Thus it happens that in cases where the inflammation is violent, before we think it possible, we have the whole mastoid process converted into a pus cavity. It has often been said that the ordinary acute otitis media will, if attended to at once, never lead to serious complications. This is, however, not my experience. Sometimes serious complications will ensue in spite of all that we do.

Dural abscesses, the essayist stated, may exist some time without distinct symptoms. Only seven weeks ago I had a case of acute otitis media following influenza, which was very serious from the start. The temperature was never lower than 102 and the discharge was profuse for weeks. At the same time severe pain developed over the temporal bone. All the symptoms of a mastoiditis were present when, after six weeks' treatment, I opened the temporal bone. It was one large cavity filled with pus and granulations. When cleansed thoroughly I found that a small amount of pus had collected below the posterior and anterior wall of the enormously large antrum and the dura-mater. About five or six drops of pus escaped from this location, after enlarging the pin-hole opening which existed in the bone. The patient made an excellent recovery. I believe that in a case where we have mastoiditis, which is not primary, we can not cure it by simply making an incision through the integument, but only by entering the cavity and removing the pus. But in cases of primary or secondary periostitis of the mastoid process, without any accumulation of pus, I do not see why Wilde's incision should not suffice.

In regard to that part of the paper referring to cerebral abscesses as one of the possible complications, I will report one case which I had, only a few months ago, under my observation. It is interesting on account of the enormous size of the cerebral abscess. The patient had been in a comatose condition for three weeks when received at the Cincinnafi City Hos. pital. The history was meager, but it was found that there had been suppuration for some time previous. It was stated by relatives that a little piece of bone had been discharged into the auditory canal. There was a profuse purulent discharge from the right ear which was exceedingly offensive; caries of the attic could be determined. Upon opening the antrum $I$ found it filled with pus and granulations, and the tegmen tympani bare; in this a very small perforation was found, through which a few drops of pus escaped. This opening was gradually enlarged, when a great amount of pus, very offensive and of a green color, came out. The abscess cavity was large. After establishing thorough drainage the wound was closed. The patient recovered from the comatose condition, but died in thirty-six hours after the operation. It was the largest abscess that any one connected with the hospital ever saw, being about the size of a small orange and occupying the right temporo-sphenoidal lobe. The brain tissue within one-fourth and one-half inch of the walls of the abscess cavity was softened and discolored.

Dr. Myles--My experience has taught me that nothing but frequent demonstration of these practical anatomic points will fix them permanently in the mind. The topography of the antrum and mastoid varies in different cases, and in different ages. The doctor has presented some remarkable specimens, but I would have presented them in a little different way. I leave all the external landmarks on the specimens, that will not be detrimental to them, as I find that in this way the mind is better enabled to grasp the situation. A complete analysis of the relation of every structure is essential in the proper comprehension, and in the teaching of this department. It is well to instruct the student so that he can operate safely, and without penetrating the lateral sinus under any topographic condition. One good rule in operating is to cut carefully and deliberately, and to start by chiseling a broad beveled opening rather than a round narrow hole. If he clings closely to the auditory canal, and cuts in a spiral direction upward, inward and forward, he will reach the antrum quickly and surely. If you wish to be a little more direct, you may start a little higher in the suprameatal triangle and chisel directly inward. The chief thing to avoid is injury to the facial nerve, which usually comes outward near the junction of the floor of the antrum and the posterior superior walls of the tympanic cavity.

In regard to children and to the paper of Dr. Roy, a great many cases of swelling posterior to the ear, and over the antrum or mastoid, can be cured by a Wilde's incision. In that class of cases where there is no pus discharge in the canal, it is usually due to periostitis or caries either of traumatic or tubercular origin, or extends through a small venous aperture from the antrum, and in certain cases the pus burrows from the attic along the external auditory canal, and causes local abscess on the roughened area of the mastoid process. A great many of these cases recover after simply incising and draining, without curettage or interference with the middle ear.

Dr. Frank Allport, Minneapolis, Minn.-1 must take issue with what has been said concerning the unnecessary frequency of such operations. My own tendencies are somewhat conservative, especially where serious operative procedures are contemplated, but in this line of cases I emphatically believe we do not operate enough. The history of the mastoid operation, and now the history of operations for otitic brain abscess, leads us through the uncertain maze of the past, when such procedures were regarded with mingled feelings of awe and aversion, to the triumphant place now universally accorded them in the modern annals of surgery, and I predict for them, and especially the latter, a much higher and more exalted position than that at present possessed. The time will come when the human brain will no longer remain a terra incognita, but will be successfully explored by the progressive modern surgeon.

No man should venture upon such surgery unless thoroughly prepared therefor by accurate anatomic, topographic and pathologic studies, both theoretic and practical; but when thus fortified, and then brought face to face with a case of this character, he should not hesitate. In a correctly recognized case of brain abscess there is no other course to pursue, and the patient may as well die from an unsuccessful operation as from a timorous and too conservative therapeusis. It is astonishing how much interference the brain will endure, as is instanced by a case I will briefly recite: The patient was a man injured in a trolley car accident, producing a basal fracture and bleeding from the ears, followed by some discharge. Cerebral symptoms ensued, and I was called to diagnose a mastoid abscess, which I could not do. The consulting physicians and myself thereupon diagnosed a probable brain 
abscess and the patient was accordingly trephined. Thorough exploration was made in many directions, starting from the temporo-sphenoidal lobe, but no pus was found. The wound was closed and death speedily expected; but the patient quickly and completely recovered, whether from relief of pressure we do not know. So, death need not always be expected, even when an operation is made and no pus found. I do not repeat this instance to encourage reckless operating, but to endeavor to dissipate the reluctant attitude assumed by surgeons when cerebral operations are mentioned.

It is not necessary for a man to be an aurist to open the mastoid process. Any surgeon properly qualified may undertake it, but it should be remembered that the operation has developed from a mere gimlet-hole opening or vent to a thorough removal of the outside mastoid shell, and a conscientious following up of every pus avenue, no matter where it may lead. We never know, therefore, when we open a mastoid process, what may be the ultimate issue involved, and we should be prepared to meet and care for any and all emergencies; and to treat pus deposits in this locality upon the same general principles observed in other portions of the body.

In regard to the method of operating, I prefer the dental engine, with fresh, sharp burs. It is gentler, quicker and smoother than the chisel, and the field of operation can be observed with great accuracy.

Concerning Wilde's incision, I believe it to be indicated but seldom, but when used, unless a pus deposit upon the outside of the mastoid is evident, the incision should be made in the inner, upper and posterior portion of the meatus, as the mas. toid cells are here much more in evidence than at the hard external mastoid plate. At best, however, the Wilde's incision is usually but a temporizing procedure, through which valuable time is lost; and still I can not say I have never seen benefit ensue through its agency.

Dr. Cline-I have seen some remarkable cases of mastoid disease and $I$ have been fully persuaded that too many extensive operations are performed on these cases. I was called to see a man seventy years old who had a discharge of pus from his ear for ten weeks, and for eight days and nights could not lie down on account of pain. On making Wilde's incision the knife passed into the bone, which seemed soft and pulpy. Pain subsided immediately and he was able to lie down. A draining tent was introduced, and the second day there was a free dis charge of pus. I prescribed iodid of potassium and he made a rapid recovery without any scraping or chiseling away of the bone.

In another case the ear had been discharging for fourteen weeks. The ear was very painful and over the mastoid the tissue was very much swollen and of a very dark purple color. His condition was such that, with his previous history, I considered an operation was indispensable. I directed him to return home and send for a surgeon in his town and have the operation performed at once. The doctor who took him in eharge decided to wait a day or two before operating. He put him on ten-grain doses of iodid of potassium every two hours. The patient began to improve, in three days the pain had disappeared and in seven days the discharge from the ear ceased, and since then (two years) he has had no further trouble.

I might detail other cases but these will suffice for the plea for conservative, early extensive operating and the free use of the iodids in these cases.

Dr. T. H. Shasted, Galesburg, Ill.-I am inclined to believe that Politzer's ideas on this point are correct. He says that in the chronic mastoid troubles the thorough operation should be done. The antrum should be opened, and, if then found necessary, the tympanum also. In acute cases, however, the thorough operation is seldom necessary. The abscess usually lies in the vertical portion of the process and does not, as a rule, commu nicate with the antrum. If in such cases, we cut down to the antrum, we shall infect those parts when there is no necessity for it. In those cases where the pus bursts through the cortex spontaneously, there is very little to be done. We can easily remove whatever comes away and that is about all there is to be done. It is seldom necessary in such cases to make a thorough operation on the ground of preventing chronic fistulous discharge. I have frequently seen such cases and they almost invariably do well in the course of two or three weeks at the longest.

Dr. Roy's case reminds me of my own, that of a lad about 18 years of age. There was no suppuration from the ear, nor sign or history of former suppuration. At the operation I found very little pus, but some carious bone. I had the case diagnosed as primary mastoiditis. But afterward I was somewhat surprised to receive from his parents a history decidedly different from what they had first given me. He had had a slight discharge of short duration some years before. No doubt in all these cases there has been previous tympanic trouble, of which the membranic signs have been effaced and the history forgotten.

Dr. B. Alexander Randall, Philadelphia, Pa.--I wish merely to refer to the point already made that these mastoid and tympanic cases are the hot-bed of tuberculosis. Cases that are not primarily tubercular are here transformed, and we have the tubercular infection passed into the whole body.

While I do not believe in Wilde's incision, I think the whole matter can be set down as largely one of technique; and the way in which we proceed will depend upon our instruments.

Dr. Edward J. Bernstein, Baltimore, Md.--I do not believe that the relative impunity with which one can enter the brain constitutes any warrant for the frequency with which some undertake these operations. I have operated a number of times with a good percentage of successes; on the other hand I have had cases, of late, where everything indicated the necessity for operation, and I plead with the patient for the necessary permission, but owing to their persistent refusal, I was forced to keep to less radical means. Many of these latter patients recovered and are apparently well. They are not safe from recurrences, as are those who submit to the radical procedure, but an immunity from attack for one or two years speaks well for their side of the question. I have never used Wilde's incision, because Leiter's coil, mercurial ointment, and other local applications have always served me where the regular mastoid operation was not indicated.

\section{ELECTROLYSIS FOR THE REDUCTION OF SPURS OF THE NASAL SEPTUM.}

Read in the Section on Laryngology and Otology, at the Forty-seventh Annual Meeting of the American Medical Association, held at Atlanta, Ga., May 5-8, 1896.

BY W. E. CASSELBERRY, M.D.

Professor of 'Laryugology and Rhinology in Northwestern University Medical School (chicago Medical College); Laryngologist and Rhinologist to St. Luke's Hospital; Laryng. ologist to Wesley Hospital, etc. CHICAGO, ILL.

A year ago I gave the results of recent experience with electrolysis for the reduction of spurs of the nasal septum, in a paper read before the section of Laryngology and Otology of the American Medical Association, ${ }^{1}$ and later in another paper before the American Laryngological Association, ${ }^{2}$ and it is not my purpose in this supplementary report to repeat the technical details of the procedure or to recite cases at length, but simply to formulate conclusions bearing upon the exact limitations of this method.

The bibliography having been presented in previous papers, will be omitted at this time; suffice it to

J JOURAL OF THF AMERICAX MEDICAL ASSOCIATION, 1895.

- Transuctions of the Am. Laryn. Assoc., 189; ; Xew York Medical Journal Ang. $31.189 \overline{5}$. 